\title{
Let's go dynamic - a quick tour through $2+1$ lattice simulations to understand chiral symmetry breaking
}

\author{
Véronique Bernard \\ Institut de Physique Nucléaire, CNRS/Univ. Paris-Sud 11 (UMR 8608), \\ 91406 Orsay Cedex, France \\ E-mail: bernardein2p3.fr \\ Sébastien Descotes-Genon* and Guillaume Toucas \\ Laboratoire de Physique Théorique, CNRS/Univ. Paris-Sud 11 (UMR 8627), \\ 91405 Orsay Cedex, France \\ E-mail: descoteseth.u-psud.frand toucaseth.u-psud.fr
}

Several lattice collaborations performing simulations with $2+1$ light dynamical quarks have experienced difficulties in fitting their data with standard $N_{f}=3$ chiral expansions at next-to-leading order, yielding low values of the quark condensate and/or the decay constant in the $N_{f}=3$ chiral limit. A reordering of these expansions seems required to analyse these data in a consistent way. We discuss such a reordering, known as Resummed Chiral Perturbation Theory and show that it provides a good fit of the recent results of two lattice collaborations (PACS-CS and $\mathrm{RBC} / \mathrm{UKQCD}$ ) concerning pion and kaon decay constants and masses as well as $K_{\ell 3}$ form factors. We describe the emerging picture for the pattern of chiral symmetry breaking, marked by a strong dependence of the observables on the strange quark mass and thus a significant difference between chiral symmetry breaking in the $N_{f}=2$ and $N_{f}=3$ chiral limits. ${ }^{\dagger}$

The XXVIII International Symposium on Lattice Filed Theory

June 14-19,2010

Villasimius, Sardinia Italy

\footnotetext{
* Speaker.

†Work supported in part by EU Contract No. MRTN-CT-2006-035482, "FLAVIAnet".
} 
Recent improvements in lattice simulations have paved the way for unquenched simulations with three light dynamical (or sea) quarks, enabling one to compare their results with the effective description of the light hadronic degrees of freedom, i.e., Chiral Perturbation Theory $(\chi \mathrm{PT})$. It turns out that several collaborations performing simulations with $2+1$ dynamical quarks reported difficulties when fitting their data with $N_{f}=3$ next-to-leading-order (NLO) chiral expansions for pseudoscalar masses, decay constants, and $K_{\ell 3}$ form factors [1,2]. Moreover, some chiral order parameters (in particular the quark condensate and the decay constant) were seen to decrease significantly when one moved from the $N_{f}=2$ chiral limit (where $m_{u}, m_{d} \rightarrow 0$ but $m_{s}$ is kept at its physical values) to the $N_{f}=3$ chiral limit (where $m_{u}, m_{d}, m_{s} \rightarrow 0$ ) [1, 3, 4].

It was advocated in refs. [5, 6] that such a situation could indeed occur in the low-energy dynamics of QCD, where the strange quark may play a very special role, due to its light mass of order $O\left(\Lambda_{Q C D}\right)$. Significant vacuum fluctuations of $s \bar{s}$ pairs may lead to instabilities in the convergence of $\chi \mathrm{PT}$ expansions, i.e., there is a numerical competition between the terms considered as LO and NLO in the chiral counting. This effect would be related to a large violation of the Zweig rule in the scalar sector, indicated by values of the $O\left(p^{4}\right)$ LECs $L_{4}$ and $L_{6}$ significantly different from specific ("critical") values. In ref. [6], Resummed Chiral Perturbation Theory (Re $\chi$ PT) was introduced to cope with such a numerical competition, and it was applied to pseudoscalar masses and decay constants, as well as $\pi \pi$ and $\pi K$ scatterings $[6,7]$. We present here results of recent fits to lattice data on pseudoscalar masses, decay constants, and $K_{\ell 3}$ form factors within this framework [8], performed in order to understand if the difficulties experienced by lattice collaborations are due to instabilities in chiral expansions, and to determine the pattern of $N_{f}=3$ chiral symmetry breaking.

\section{Resummed Chiral Perturbation Theory}

In Re $\chi$ PT, only some ("good") observables are assumed to have convergent expansions, in the sense that the sum of LO and NLO terms is large compared to the remaining part of the series. These quantities with convergent chiral series are those taken as a starting point to build chiral perturbation theory (using the formalism of the generating functional), namely the QCD correlators of axial and vector currents as well as pseudoscalar and scalar densities. From the chiral point of view, the convergence is expected to be good only away from the singularities (poles, cuts...) corresponding to resonances and channel openings. Observables involving pseudoscalar mesons as external states are obtained by applying the LSZ reduction formula on correlators involving axial currents, which yields factors of the relevant pseudoscalar decay constants.

We treat a "good" observable in the following way : $a$ ) we take the chiral expansion of the observable in terms of the couplings of the effective Lagrangian (low-energy constants, or LECs: $\left.B_{0}, F_{0}, L_{i} \ldots\right), b$ ) we replace the pseudoscalar masses at leading order by the physical ones only when physical arguments indicate that the convergence of the series will be improved (in practice to ensure the nonanalytic structures imposed by unitarity to be located at the physical poles, thresholds...) and $c$ ) we keep track of the remainders (collecting NNLO contributions and higher orders) explicitly and treat the resulting chiral expansions as algebraic identities, without performing further expansions. Following this procedure (and taking the isospin limit $m_{u}=m_{d}=m$ ), observables can be expressed in terms of LO quantities:

$$
X(3)=2 m \Sigma(3) / F_{\pi}^{2} M_{\pi}^{2}, \quad Z(3)=F^{2}(3) / F_{\pi}^{2}, \quad r=m_{s} / m,
$$


as well as NLO LECs and remainders. The first two quantities in eq. (1.1) are of particular relevance, since they express two main order parameters of $N_{f}=3$ chiral symmetry breaking, the quark condensate and the pseudoscalar decay constant, in physical units. They also assess the saturation of the chiral expansion of $F_{\pi}^{2} M_{\pi}^{2}$ and $F_{\pi}^{2}$ by their leading order. The third quantity measures the relative size of the quark masses in a framework where the strange quark is supposed to play a peculiar role in the chiral structure of QCD vacuum.

We introduce also $Y(3)=2 m B_{0} / M_{\pi}^{2}=X(3) / Z(3)$, which assesses the saturation of the chiral expansion of the pion mass by its leading order. Exploiting the fact that some quantities describing the dynamics of pseudoscalar mesons are well measured, we can invert the relationships between these observables and LECs to express the $O\left(p^{4}\right)$ LECs in terms of masses, decay constants, radii of form factors, scattering parameters... as well as the three leading-order parameters described in eq. (1.1) and the higher-order remainders associated to each observable, assumed to be small. The "good" quantities related to $F_{\pi}^{2}, F_{K}^{2}, F_{\pi}^{2} M_{\pi}^{2}, F_{K}^{2} M_{K}^{2}$ and the pion electromagnetic radius led to such expressions for the NLO low-energy constants $L_{4}, L_{5}, L_{6}, L_{8}$ and $L_{9}$. For instance, from the expansion of $F_{\pi}^{2}$ and $F_{K}^{2}$, we can extract $L_{5}$ and $L_{4}$, the latter being:

$$
Y(3)\left[L_{4}^{r}(\mu)-\hat{L}_{4}^{r}(\mu)\right]=\frac{1}{8(r+2)} \frac{F_{\pi}^{2}}{M_{\pi}^{2}}\left[1-\frac{2}{r-1}\left(\frac{F_{K}^{2}}{F_{\pi}^{2}}-1\right)-Z(3)-e\right],
$$

where $\hat{L}_{4}$ is a known numerical function of the LO parameters eq. (1.1) collecting chiral logarithms coming from tadpole contributions, whereas $e$ combines higher-order remainders attached to the expansion of $F_{\pi}^{2}$ and $F_{K}^{2}$. These expressions can then be exploited in the chiral expansions of other "good" observables, in order to express them in terms of LO quantities and remainders only, and to extract information on the latter from experiment or lattice results.

\section{2. $K_{\ell 3}$ decays}

Among the quantities that can be determined from lattice simulations, one can single out the $K_{\ell 3}$ form factors defined as [10]:

$$
\sqrt{2}\left\langle K^{+}\left|\bar{u} \gamma_{\mu} s\right| \pi^{0}\right\rangle=\left(p^{\prime}+p\right)^{\mu} f_{+}(t)+\left(p^{\prime}-p\right)^{\mu} f_{-}(t), \quad f_{0}(t)=f_{+}(t)+\frac{t}{\Delta_{K \pi}} f_{-}(t) .
$$

where $\Delta_{P Q}=M_{P}^{2}-M_{Q}^{2} . f_{+}$corresponds to $P$-wave projection of the $K_{\ell 3}$ transition, whereas its $S$-wave comes from $f_{0} . F_{\pi} F_{K} f_{+}$and $F_{\pi} F_{K} f_{-}$are expected to have good convergence properties away from the singularities (opening thresholds...) and their chiral expansions can be expressed in terms of $r, X(3), Z(3)$, NLO low-energy constants $\left(L_{4}, L_{5}\right.$ and $\left.L_{9}\right)$ and remainders. Reexpressing $L_{4}$ and $L_{5}$ using the expansion of $F_{\pi}^{2}$ and $F_{K}^{2}$ yields:

$$
\begin{aligned}
& F_{\pi} F_{K} f_{+}(t)=\frac{F_{\pi}^{2}+F_{K}^{2}}{2}+\frac{3}{2}\left[t M_{K \pi}^{r}(t)+t M_{K \eta}^{r}(t)-L_{K \pi}(t)-L_{K \eta}(t)\right]+2 t L_{9}^{r}+F_{\pi} F_{K} d_{+}+t e_{+}, \quad \text { (2.2) } \\
& F_{\pi} F_{K} f_{-}(t)=\frac{F_{K}^{2}-F_{\pi}^{2}}{2}-\frac{3}{2}\left(M_{K}^{2}-M_{\pi}^{2}\right)\left[M_{K \pi}^{r}(t)+M_{K \eta}^{r}(t)\right] \\
& +\frac{1}{4} K_{K \pi}(t)\left[5\left(t-M_{\pi}^{2}-M_{K}^{2}\right)+\frac{3}{2}(r+3) M_{\pi}^{2} Y(3)\right]-\frac{1}{4} K_{K \eta}(t)\left[3\left(t-M_{\pi}^{2}-M_{K}^{2}\right)+\frac{1}{2}(r+3) M_{\pi}^{2} Y(3)\right] \\
& -2\left(M_{K}^{2}-M_{\pi}^{2}\right) L_{9}^{r}+F_{\pi} F_{K}\left(d_{-}-d_{+}\right)+t\left(e_{-}-e_{+}\right),
\end{aligned}
$$


where $L$ and $M$ are well-known functions defined from the one-loop two-propagator scalar integral, and $d_{ \pm}=O\left(m_{q}^{2}\right)$ and $e_{ \pm}=O\left(m_{q}\right)$ combine remainders from the form factors and the decay constants. We can write a similar expression for the pion electromagnetic form factor $F_{\pi}^{2} F_{V}^{\pi}$ and invert it, which providing an expression of $L_{9}$ in terms of the pion electromagnetic radius, its higher-order remainder and. This expression of $L_{9}$ can then be used in eq. (2.2) to yield the expression of $f_{+}$and $f_{-}$in terms of the three fundamental parameters in eq. (1.1), the physical values of pion and kaon decay constants and masses and the pion electromagnetic radius, and the higher-order remainders. If we assume all expansions to be saturated by their LO contribution, we recover the well-known expression for the form factors at NLO in chiral perturbation theory [10].

These expressions satisfy the Callan-Treiman theorem: in the soft-pion limit $\left(p^{\prime 2}=M_{\pi}^{2}=0\right)$, the scalar form factor at $t=\Delta_{K \pi} \equiv M_{K}^{2}-M_{\pi}^{2}$ (Callan-Treiman point) should be equal to $F_{K} / F_{\pi}$. This implies that $F_{K} F_{\pi} f_{0}\left(\Delta_{K \pi}\right)-F_{K}^{2}$ vanishes in the $N_{f}=2$ chiral limit $m_{u}=m_{d} \rightarrow 0$. There is a softkaon analog of this theorem holding at $t=\tilde{\Delta}_{K \pi} \equiv-\Delta_{K \pi}$, stating that $F_{K} F_{\pi} f_{0}\left(\tilde{\Delta}_{K \pi}\right)-F_{\pi}^{2}$ vanishes in the $N_{f}=3$ chiral limit. This provides constraints on the NNLO remainders: $d_{-}=O\left(\mathrm{~mm}_{s}\right)$, $e_{-}=O(m)$, thus $1 / r$-suppressed compared to $d_{+}$and $e_{+}$. The discrepancies from the CallanTreiman theorem(s) $\Delta_{C T}=f_{0}\left(\Delta_{K \pi}\right)-F_{K} / F_{\pi}$ and $\tilde{\Delta}_{C T}=f_{0}\left(-\Delta_{K \pi}\right)-F_{\pi} / F_{K}$ were calculated in standard $\chi$ PT at one-loop order in the isospin limit [10]: $\Delta_{C T}=-3.5 \cdot 10^{-3}, \tilde{\Delta}_{C T}=0.03$.

\section{Fits to $2+1$ lattice data}

The expression of NLO LECs in terms of $r, X(3), Z(3)$, accurately measured observables (masses, decay constants, pion electromagnetic radius) and remainders can be inserted in the chiral expansions of other observables (such as kaon form factors, or meson-meson scattering), which can be used to constrain $r, X(3)$ and $Z(3)$. For each new observable, one or several remainders are introduced, which are assumed to be small but nevertheless limit the accuracy of the chiral series. Rather than taking observables of a different type (such as scattering processes), we consider here the values for the same observables obtained from lattice simulations with 3 dynamical flavours, with different values of the quark masses $\left(\tilde{m}, \tilde{m}, \tilde{m}_{s}\right)$. We can then use the same Re $\chi$ PT expressions as in the physical case, involving known NLO LECs - which can be reexpressed in terms of the fundamental parameters eq. (1.1) as explained before - and higher-order remainders - related to the physical ones if one assumes simple scaling laws as the quark masses vary. We take data of the PACS-CS [1] and RBC/UKQCD [2] collaborations for the pion and kaon pseudoscalar decay constants and masses, adding the $K_{\ell 3}$ form factors for RBC/UKQCD. In both cases, we fit only a "subset" of the data with light quark masses and small momenta, in order to stay in a domain where Re $\chi$ PT expressions should be valid. We can thus determine what the dependence of lattice data on quark masses and momentum suggests on chiral symmetry breaking in the $N_{f}=3$ chiral limit, irrespective of assumptions on the relative size of LO and NLO in chiral expansions [8].

Our results are summarised in Table 1. The first series of rows corresponds to the outcome of the fit, whereas the lower rows are quantities computed from the results of the fit (LO LECs, NLO LECs, $N_{f}=2$ order parameters, $K_{\ell 3}$ quantities, relative fraction of LO/NLO/remainders at the minimum for several observables), and the last row is the $\chi^{2}$ per degree of freedom. $F_{K} / F_{\pi}$ (left free in our fit) comes out slightly larger (smaller) for PACS-CS (RBC/UKQCD) than its Standard Model value based on leptonic and semileptonic decays. We obtain also values of quark masses 
in good agreement with the results obtained by the two collaborations. The pseudoscalar decay constant in the $N_{f}=3$ chiral limit is found to be rather low, as well as as the quark condensate, which is reflected by the low-energy constants $L_{4}$ and $L_{6}$, both positive and showing no sign of Zweig suppression. $L_{5,8,9}$ have values in agreement with conventional estimates. On the other hand, we obtain values for $N_{f}=2$ chiral order parameters which are in agreement with a saturation of two-flavour chiral series by their LO, and with the experimental information on $\pi \pi$ scattering.

The $K_{\ell 3}$ form factor at zero momentum transfer, $f_{+}(0)=f_{0}(0)$ involves only LECs related to decay constants and masses, eq. (2.2) and is related to the determination of the CKM matrix element $\left|V_{u s}\right|$ in $K_{\ell 3}$ decays. The value obtained for $f_{0}(0)$ in our fit is somewhat larger than the values obtained from the RBC/UKQCD collaboration using different forms for the extrapolation in quark masses. The chiral expansion of $f_{+}(0)=1+f_{2}+f_{4}+\ldots$ involves the NLO contribution $f_{2}$ which is only a combination of chiral logarithms divided by $F_{0}^{2}$ :

$$
f_{2}=-\frac{3}{256 \pi^{2} F_{0}^{2}}\left[\left(M_{K}^{2}+M_{\pi}^{2}\right) h\left(\frac{M_{\pi}^{2}}{M_{K}^{2}}\right)+\left(M_{K}^{2}+M_{\eta}^{2}\right) h\left(\frac{M_{\eta}^{2}}{M_{K}^{2}}\right)\right], \quad h(x)=1+\frac{2 x}{1-x^{2}} \log x .
$$

$f_{2}$ is often said to be free from LECs and thus known precisely from Chiral Perturbation Theory, assuming that the value of $F_{0}$ is close to that of $F_{\pi}$ and can be replaced by the physical value of the pion decay constant leading to the estimate $f_{2} \simeq-0.023$. Since the difference between the two quantities is a higher order effect, one can always perform this replacement, but one has to determine how large the NNLO term $f_{4}$ can be with such a prescription. If $F_{0}$ is significantly lower than $F_{\pi}$ as indicated not only by our fit, but by other recent estimates [9], the convergence is expected to be rather slow. We advocated that correlators of vector and axial currents yield then observables with good convergence properties, selecting $F_{\pi} F_{K} f_{0}(0)$. We should then replace $F_{0}^{2}$ by $F_{\pi} F_{K}$ in eq. (3.1), as can be checked in our expression for $f_{+}$, eq. (2.2).

In the last lines of our table, we indicated for each fit the contribution from LO, NLO and remainders to pseudoscalar decay constants and masses for values of the parameters at the minimum of the fit. We can see that the series converge well on overall (remainder much smaller than $\mathrm{LO}+\mathrm{NLO}$ ), but that the LO term is far from saturating the series.

\section{Conclusion}

Recent lattice simulations with light $2+1$ dynamical fermions have encountered difficulties to fit their results for pseudoscalar masses, decay constants and form factors with chiral expansions obtained from $N_{f}=3$ Chiral Perturbation Theory at next-to-leading order. Such fits of poor quality may due to the fact that chiral series are not saturated by their leading order, so that there is a numerical competition between LO and NLO contributions. We proposed an alternative treatment of chiral expansions, Resummed Chiral Perturbation Theory, to deal with such a situation, applying it to pion and kaon masses and decay constants, as well as $K_{\ell 3}$ form factors. Our framework is compatible with the usual assumptions that chiral series of decay constants, squared masses... are saturated by their LO contribution, but it can also accommodate situations where there is a numerical competition between LO and NLO terms.

Then we turned to $2+1$ lattice simulations where these observables were obtained for several sets of quark masses: PACS-CS (decay constants and masses only) [1] and RBC/UKQCD (decay 
constants, masses, $K_{\ell 3}$ form factors) [2], restricted to light quark masses and small momenta. The fits favour values for the three quantities $X(3), Y(3), Z(3)$ smaller than 1 , with a $\chi^{2} /$ d.o.f. which ranges from fairly good to excellent. The decay constant and the quark condensate in the $N_{f}=3$ limit ( $\left.m_{u}=m_{d}=m_{s}=0\right)$ are found both small and suppressed compared to the $N_{f}=2$ case ( $m_{u}=m_{d}=0$ and $m_{s}$ physical). The low-energy constants $L_{4}$ and $L_{6}$ do not follow the Zweig rule suppression generally advocated to set them to zero, whereas the other low-energy constants $L_{5}$, $L_{8}$ and $L_{9}$ have values in good agreement with previous estimates. The ratio of quark masses $r$ remains quite close to the most simple estimate from pseudoscalar masses. $N_{f}=2$ chiral order parameters are in good agreement with the values extracted from $K_{\ell 4}$ decays. The expected numerical competition between $\mathrm{LO}$ and NLO chiral expansions indeed occurs for $F_{\pi}^{2}, F_{K}^{2}, F_{\pi}^{2} M_{\pi}^{2}$ and $F_{K}^{2} M_{K}^{2}$.

Our approach provides an alternative to the chiral expansions generally used to extrapolate lattice data. The usual expansions exhibit chiral logarithms of the form $M_{P}^{2} \log \left(M_{P}^{2} / \mu^{2}\right)$ which can prove quite troublesome to fit. For instance, the RBC/UKQCD collaboration [2] finds a better agreement of their data on decay constants with polynomial fits than with chiral series. In our treatment, these chiral logarithms always involve the LO term of the pseudoscalar masses (rather than the physical masses). Therefore, a small value of $Y(3)$ (or $B_{0}$ ) will tame the chiral logarithms in our expansion, while large contributions from NLO LECs, and in particular from $L_{4}$ and $L_{6}$, will enhance the quadratic dependence on the quark masses. Therefore, even though our expressions do include chiral logarithms, they are also able to mimic a polynomial dependence on the quark masses which cannot be reproduced in the usual treatment of chiral expansions.

Finally, let us notice that the value obtained for $f_{+}(0)$ with our fits is larger than the ones quoted by the RBC/UKQCD collaboration, relying on alternative chiral expansions for $K_{\ell 3}$ form factors. We expect this question of chiral extrapolations of lattice data to become more and more relevant for $\left|V_{u s}\right|$, considering the accuracy currently achieved in $K_{\ell 3}$ decays.

\section{References}

[1] S. Aoki et al. [PACS-CS Collaboration], Phys. Rev. D 79 (2009) 034503 [arXiv:0807.1661 [hep-lat]].

[2] C. Allton et al. [RBC-UKQCD Collaboration], Phys. Rev. D 78 (2008) 114509 [arXiv:0804.0473 [hep-lat]]; P. A. Boyle et al., Phys. Rev. Lett. 100 (2008) 141601 [arXiv:0710.5136 [hep-lat]]; P. A. Boyle et al., arXiv:1004.0886 [hep-lat].

[3] C. Bernard et al., PoS LAT2007 (2007) 090 [arXiv:0710.1118 [hep-lat]].

[4] A. Bazavov et al. [The MILC Collaboration], PoS LAT2009 (2009) 079 [arXiv:0910.3618].

[5] S. Descotes-Genon, L. Girlanda and J. Stern, JHEP 0001 (2000) 041 [hep-ph/9910537].

[6] S. Descotes-Genon and J. Stern, Phys. Lett. B 488 (2000) 274 [hep-ph/0007082]; S. Descotes-Genon, N. H. Fuchs, L. Girlanda and J. Stern, Eur. Phys. J. C 34 (2004) 201 [hep-ph/0311120].

[7] S. Descotes-Genon, Eur. Phys. J. C 52 (2007) 141 [arXiv:hep-ph/0703154].

[8] V. Bernard, S. Descotes-Genon and G. Toucas, arXiv:1009.5066 [hep-ph].

[9] J. Bijnens and I. Jemos, arXiv:0909.4477; V. Bernard and E. Passemar, JHEP 1004 (2010) 001 [arXiv:0912.3792].

[10] J. Gasser and H. Leutwyler, Nucl. Phys. B 250 (1985) 517; J. Gasser and U.-G. Meißner, Nucl. Phys. B 357 (1991) 90; J. Bijnens and P. Talavera, JHEP 0203 (2002) 046 [hep-ph/0203049]. 


\begin{tabular}{|c|c|c|}
\hline & PACS - CS Subset & RBC/UKQCD Subset \\
\hline$r$ & $26.5 \pm 2.3$ & $23.2 \pm 1.5$ \\
\hline$X(3)$ & $0.59 \pm 0.21$ & $0.20 \pm 0.14$ \\
\hline$Y(3)$ & $0.90 \pm 0.22$ & $0.43 \pm 0.30$ \\
\hline$Z(3)$ & $0.66 \pm 0.09$ & $0.46 \pm 0.04$ \\
\hline$F_{K} / F_{\pi}$ & $1.237 \pm 0.025$ & $1.148 \pm 0.015$ \\
\hline$m_{s}(2 \mathrm{GeV})[\mathrm{MeV}]$ & $70 \pm 4$ & 107 (fixed) \\
\hline$m(2 \mathrm{GeV})[\mathrm{MeV}]$ & $2.6 \pm 0.3$ & $4.6 \pm 0.3$ \\
\hline$B_{0}(2 \mathrm{GeV})[\mathrm{GeV}]$ & $3.34 \pm 1.18$ & $0.92 \pm 0.67$ \\
\hline$F_{0}[\mathrm{MeV}]$ & $74.8 \pm 4.9$ & $62.2 \pm 2.5$ \\
\hline$L_{4}\left(M_{\rho}\right) \cdot 10^{3}$ & $-0.1 \pm 0.2$ & $2.4 \pm 2.0$ \\
\hline$L_{5}\left(M_{\rho}\right) \cdot 10^{3}$ & $1.8 \pm 0.4$ & $1.8 \pm 1.6$ \\
\hline$L_{6}\left(M_{\rho}\right) \cdot 10^{3}$ & $0.1 \pm 0.4$ & $4.7 \pm 7.1$ \\
\hline$L_{8}\left(M_{\rho}\right) \cdot 10^{3}$ & $0.8 \pm 0.7$ & $4.4 \pm 7.1$ \\
\hline$L_{9}\left(M_{\rho}\right) \cdot 10^{3}$ & $x$ & $4.4 \pm 2.8$ \\
\hline$X(2)$ & $0.90 \pm 0.01$ & $0.90 \pm 0.02$ \\
\hline$Y(2)$ & $1.04 \pm 0.02$ & $1.00 \pm 0.03$ \\
\hline$Z(2)$ & $0.87 \pm 0.02$ & $0.90 \pm 0.02$ \\
\hline$B(2 \mathrm{GeV})[\mathrm{GeV}]$ & $3.83 \pm 0.50$ & $2.09 \pm 0.19$ \\
\hline$F[\mathrm{MeV}]$ & $85.8 \pm 0.7$ & $87.7 \pm 0.8$ \\
\hline$\Sigma / \Sigma_{0}$ & $1.51 \pm 0.51$ & $4.52 \pm 2.83$ \\
\hline$B / B_{0}$ & $1.15 \pm 0.26$ & $2.28 \pm 1.39$ \\
\hline$F / F_{0}$ & $1.15 \pm 0.08$ & $1.41 \pm 0.06$ \\
\hline$f_{+}(0)$ & $1.004 \pm 0.149$ & $0.985 \pm 0.008$ \\
\hline$\Delta_{C T} \cdot 10^{3}$ & $x$ & $-0.2 \pm 12.1$ \\
\hline$\Delta_{C T}^{\prime} \cdot 10^{3}$ & $x$ & $-126 \pm 104$ \\
\hline$F_{\pi}^{2}$ & $0.66+0.22+0.12$ & $0.45+0.69-0.14$ \\
\hline$F_{K}^{2}$ & $0.44+0.48+0.08$ & $0.34+0.76-0.10$ \\
\hline$F_{\pi}^{2} M_{\pi}^{2}$ & $0.60+0.30+0.10$ & $0.20+0.95-0.15$ \\
\hline$F_{K}^{2} M_{K}^{2}$ & $0.42+0.50+0.08$ & $0.14+0.97-0.11$ \\
\hline$F_{\pi} F_{K} f_{+}(0)$ & $\times$ & $0.40+0.75-0.15$ \\
\hline$\chi^{2} / N$ & $0.9 / 3$ & $4.4 / 8$ \\
\hline
\end{tabular}

Table 1: Results of fits performed on the data from the PACS-CS [1] and RBC/UKQCD [2] collaborations on pseudoscalar masses and decay constants, adding $K_{\ell 3}$ form factors in the case of RBC/UKQCD. We considered only data with light pions (Subset) and only statistical errors are shown. The strange quark mass was not fitted in the RBC/UKQCD due to the limited number of points, but fixed to the value obtained by the lattice collaboration. The LEC $L_{9}$ (and the deviations from Callan-Treiman relations) could not be determined in the PACS-CS case due to the absence of $K_{\ell 3}$ data. The penultimate set of rows collects the relative fractions of LO/NLO/remainders for decay constants, masses and $K_{\ell 3}$ form factor at vanishing transfer momentum (for RBC/UKQCD) at the minimum. 